Commun. Fac. Sci. Univ. Ank. Ser. A1 Math. Stat.

Volume 68, Number 2, Pages 1647-1652(2019)

DOI: $10.31801 /$ cfsuasmas.420820

ISSN 1303-5991 E-ISSN 2618-6470

http://communications.science.ankara.edu.tr/index.php?series=A

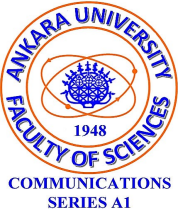

\title{
CONVOLUTION PROPERTIES FOR SALAGEAN-TYPE ANALYTIC FUNCTIONS DEFINED BY q-DIFFERENCE OPERATOR
}

\author{
ASENA ÇETINKAYA
}

\begin{abstract}
In this paper, we define Salagean-type analytic functions by using concept of $q$-derivative operator. We investigate convolution properties and coefficient estimates for Salagean-type analytic functions denoted by $\mathcal{S}_{q}^{m, \lambda}[A, B]$
\end{abstract}

\section{INTRODUCTION}

Let $\mathcal{A}$ be the class of functions $f$ defined by

$$
f(z)=z+\sum_{n=2}^{\infty} a_{n} z^{n}
$$

that are analytic in the open unit disc $U=\{z:|z|<1\}$ and $\Omega$ be the family of functions $w$ which are analytic in $U$ and satisfy the conditions $w(0)=0,|w(z)|<1$ for all $z \in U$. If $f_{1}$ and $f_{2}$ are analytic functions in $U$, then we say that $f_{1}$ is subordinate to $f_{2}$ written as $f_{1} \prec f_{2}$ if there exists a Schwarz function $w \in \Omega$ such that $f_{1}(z)=f_{2}(w(z)), z \in U$. We also note that if $f_{2}$ univalent in $U$, then $f_{1} \prec f_{2}$ if and only if $f_{1}(0)=f_{2}(0), f_{1}(U) \subset f_{2}(U)$ (see [5]).

Let $f_{1}(z)=z+\sum_{n=2}^{\infty} a_{n} z^{n}$ and $f_{2}(z)=z+\sum_{n=2}^{\infty} b_{n} z^{n}$ be elements in $\mathcal{A}$. Then the Hadamard product or convolution of these functions is defined by

$$
f_{1}(z) * f_{2}(z)=z+\sum_{n=2}^{\infty} a_{n} b_{n} z^{n} .
$$

Next, for arbitrary fixed numbers $A, B,-1 \leq B<A \leq 1$, denote by $\mathcal{P}[A, B]$ the family of functions $p(z)=1+p_{1} z+p_{2} z^{2}+\cdots$, analytic in $U$ such that $p \in \mathcal{P}[A, B]$

Received by the editors: May 03, 2018; Accepted: November 16, 2018.

2010 Mathematics Subject Classification. 30C45.

Key words and phrases. Salagean differential operator, $q$-difference operator, convolution, coefficient estimate.

(C)2019 Ankara University Communications Faculty of Sciences University of Ankara-Series A1 Mathematics and Statistics 
if and only if

$$
p(z)=\frac{1+A w(z)}{1+B w(z)}
$$

for some functions $w \in \Omega$ and every $z \in U$. This class was introduced by Janowski [8].

In 1909 and 1910 Jackson [6, 7] initiated a study of $q$-difference operator $D_{q}$ defined by

$$
D_{q} f(z)=\frac{f(z)-f(q z)}{(1-q) z} \quad \text { for } \quad z \in B \backslash\{0\},
$$

where $B$ is a subset of complex plane $\mathbb{C}$, called $q$-geometric set if $q z \in B$, whenever $z \in B$. Obviously, $D_{q} f(z) \rightarrow f^{\prime}(z)$ as $q \rightarrow 1^{-}$. The $q$-difference operator (2) is also called Jackson $q$-difference operator. Note that such an operator plays an important role in the theory of hypergeometric series and quantum physics (see for instance [1, 3, 4, 9]).

Since

$$
D_{q} z^{n}=\frac{1-q^{n}}{1-q} z^{n-1}=[n]_{q} z^{n-1},
$$

where $[n]_{q}=\frac{1-q^{n}}{1-q}$, it follows that for any $f \in \mathcal{A}$, we have

$$
D_{q} f(z)=1+\sum_{n=2}^{\infty}[n]_{q} a_{n} z^{n-1},
$$

where $q \in(0,1)$. Clearly, as $q \rightarrow 1^{-},[n]_{q} \rightarrow n$. For notations, one may refer to 4 .

The Salagean differential operator $R^{m}$ was introduced by Salagean [10] in 1998. Since then, many mathematicians used the idea of Salagean differential operator in their papers (see [2]). $q$-Salagean differential operator is defined as below:

Definition 1. The q-analogue of Salagean differential operator $R_{q}^{m} f(z): \mathcal{A} \rightarrow \mathcal{A}$ is formed by

$$
\begin{aligned}
R_{q}^{0} f(z) & =f(z) \\
R_{q}^{1} f(z) & =z D_{q}(f(z)) \\
\vdots & \\
R_{q}^{m} f(z) & =z D_{q}^{1}\left(R_{q}^{m-1} f(z)\right) .
\end{aligned}
$$

From definition $R_{q}^{m} f(z)$, we obtain

$$
R_{q}^{m} f(z)=z+\sum_{n=2}^{\infty}[n]_{q}^{m} a_{n} z^{n},
$$

where $[n]_{q}^{m}=\left(\frac{1-q^{n}}{1-q}\right)^{m}, q \in(0,1), m \in \mathbb{N}$. Clearly, as $q \rightarrow 1^{-}$, the equation (3) reduces to Salagean differential operator. 
Motivated by $q$-Salagean differential operator, we define the class of Salageantype analytic functions denoted by $\mathcal{S}_{q}^{m, \lambda}[A, B]$.

Definition 2. A function $f \in \mathcal{A}$ is said to be in the class $\mathcal{S}_{q}^{m, \lambda}[A, B]$ such that

$$
1+\frac{e^{i \lambda}}{\cos \lambda}\left(\frac{R_{q}^{m+1} f(z)}{R_{q}^{m} f(z)}-1\right) \prec \frac{1+A z}{1+B z}
$$

where $q \in(0,1),|\lambda|<\frac{\pi}{2}, m \in \mathbb{N}, z \in U$.

Also, we note that $\mathcal{C}_{q}^{m, \lambda}[A, B]$ is the class of functions $f \in \mathcal{A}$ satisfying $z D_{q} f \in$ $\mathcal{S}_{q}^{m, \lambda}[A, B]$.

In this paper, we investigate the necessary and sufficient convolution conditions and coefficient estimates for the class $\mathcal{S}_{q}^{m, \lambda}[A, B]$ associated with the $q$-derivative operator.

\section{Main Results}

We first begin with necessary and sufficient convolution conditions of our class $\mathcal{S}_{q}^{m, \lambda}[A, B]$.

Theorem 3. The function $f$ defined by (1) is in the class $\mathcal{S}_{q}^{m, \lambda}[A, B]$ if and only if

$$
\frac{1}{z}\left[R_{q}^{m} f(z) * \frac{z-L q z^{2}}{(1-z)(1-q z)}\right] \neq 0
$$

for all $L=\frac{e^{-i \theta}+(A-B) \cos \lambda e^{-i \lambda}+B}{(A-B) \cos \lambda e^{-i \lambda}}$, where $\theta \in[0,2 \pi], q \in(0,1),|\lambda|<\frac{\pi}{2}$ and also $L=1$.

Proof. First suppose $f \in \mathcal{S}_{q}^{m, \lambda}[A, B]$, then we have

$$
1+\frac{e^{i \lambda}}{\cos \lambda}\left(\frac{R_{q}^{m+1} f(z)}{R_{q}^{m} f(z)}-1\right) \prec \frac{1+A z}{1+B z}
$$

therefore we get

$$
\frac{R_{q}^{m+1} f(z)}{R_{q}^{m} f(z)} \prec \frac{1+\left((A-B) \cos \lambda e^{-i \lambda}+B\right) z}{1+B z} .
$$

Since the function from the left-hand side of the subordination is analytic in $U$, it follows $f(z) \neq 0, z \in U^{*}=U \backslash\{0\}$; that is, $\frac{1}{z} f(z) \neq 0$ and this is equivalent to the fact that (4) holds for $L=1$. From (6) according to the subordination of two analytic functions, we say that there exists a function $w$ analytic in $U$ with $w(0)=0,|w(z)|<1$ such that

$$
\frac{R_{q}^{m+1} f(z)}{R_{q}^{m} f(z)}=\frac{1+\left((A-B) \cos \lambda e^{-i \lambda}+B\right) w(z)}{1+B w(z)}
$$


which is equivalent to

$$
\frac{R_{q}^{m+1} f(z)}{R_{q}^{m} f(z)} \neq \frac{1+\left((A-B) \cos \lambda e^{-i \lambda}+B\right) e^{i \theta}}{1+B e^{i \theta}}
$$

Or

$$
\frac{1}{z}\left[\left(1+B e^{i \theta}\right) R_{q}^{m+1} f(z)-\left(1+\left((A-B) \cos \lambda e^{-i \lambda}+B\right) e^{i \theta}\right) R_{q}^{m} f(z)\right] \neq 0 .
$$

Since

$$
\begin{gathered}
R_{q}^{m} f(z) * \frac{z}{1-z}=R_{q}^{m} f(z), \\
R_{q}^{m} f(z) * \frac{z}{(1-z)(1-q z)}=R_{q}^{m+1} f(z),
\end{gathered}
$$

we may write $(9)$ as

$$
\frac{1}{z}\left[R_{q}^{m} f(z) *\left(\frac{\left(1+B e^{i \theta}\right) z}{(1-z)(1-q z)}-\frac{\left(1+\left((A-B) \cos \lambda e^{-i \lambda}+B\right) e^{i \theta}\right) z}{(1-z)}\right)\right] \neq 0 .
$$

Therefore we obtain

$$
\frac{\left((B-A) \cos \lambda e^{-i \lambda}\right) e^{i \theta}}{z}\left[R_{q}^{m} f(z) * \frac{z-\frac{e^{-i \theta}+(A-B) \cos \lambda e^{-i \lambda}+B}{(A-B) \cos \lambda e^{-i \lambda}} q z^{2}}{(1-z)(1-q z)}\right] \neq 0,
$$

which leads to (4) and the necessary part of Theorem 3.

Conversely, because assumption (4) holds for $L=1$, it follows that $\frac{1}{z} f(z) \neq 0$ for all $z \in U$; hence, the function $\varphi(z)=1+\frac{e^{i \lambda}}{\cos \lambda}\left(\frac{R_{q}^{m+1} f(z)}{R_{q}^{m} f(z)}-1\right)$ is analytic in $U$. Since it was shown in the first part of the proof that assumption (4) is equivalent to (8), we obtain that

$$
\frac{R_{q}^{m+1} f(z)}{R_{q}^{m} f(z)} \neq \frac{1+\left((A-B) \cos \lambda e^{-i \lambda}+B\right) e^{i \theta}}{1+B e^{i \theta}}
$$

and if we denote

$$
\psi(z)=\frac{1+\left((A-B) \cos \lambda e^{-i \lambda}+B\right) z}{1+B z},
$$

relation (11) shows that $\varphi(U) \cap \psi(U)=\emptyset$. Thus, the simply connected domain $\varphi(U)$ is included in a connected component of $C \backslash \psi(\partial U)$. From here, using the fact that $\varphi(0)=\psi(0)$ together with the univalence of the function $\psi$, it follows that $\varphi(z) \prec \psi(z)$, which represents in fact subordination (6); that is, $f \in \mathcal{S}_{q}^{m, \lambda}[A, B]$. This completes the proof of Theorem 3 .

Taking $q \rightarrow 1^{-}$in Theorem 3, we obtain the following result.

Corollary 4. The function $f$ defined by (1) is in the class $\mathcal{S}^{m, \lambda}[A, B]$ if and only if

$$
\frac{1}{z}\left[R^{m} f(z) * \frac{z-L z^{2}}{(1-z)^{2}}\right] \neq 0
$$


for all $L=\frac{e^{-i \theta}+(A-B) \cos \lambda e^{-i \lambda}+B}{(A-B) \cos \lambda e^{-i \lambda}}$, where $\theta \in[0,2 \pi],|\lambda|<\frac{\pi}{2}$ and also $L=1$.

Theorem 5. A necessary and sufficient condition for the function $f$ defined by (1) to be in the class $\mathcal{S}_{q}^{m, \lambda}[A, B]$ is that

$$
1-\sum_{n=2}^{\infty}[n]_{q}^{m} \frac{[n]_{q}\left(e^{-i \theta}+B\right)-e^{-i \theta}+(B-A) \cos \lambda e^{-i \lambda}-B}{(A-B) \cos \lambda e^{-i \lambda}} a_{n} z^{n-1} \neq 0 .
$$

Proof. From Theorem $3, f \in \mathcal{S}_{q}^{m, \lambda}[A, B]$ if and only if

$$
\frac{1}{z}\left[R_{q}^{m} f(z) * \frac{z-L q z^{2}}{(1-z)(1-q z)}\right] \neq 0
$$

for all $L=\frac{e^{-i \theta}+(A-B) \cos \lambda e^{-i \lambda}+B}{(A-B) \cos \lambda e^{-i \lambda}}$ and also $L=1$. The left-hand side of 15 can be written as

$$
\begin{aligned}
& \frac{1}{z}\left[R_{q}^{m} f(z) *\left(\frac{z}{(1-z)(1-q z)}-\frac{L q z^{2}}{(1-z)(1-q z)}\right)\right] \\
& =\frac{1}{z}\left\{R_{q}^{m+1} f(z)-L\left[R_{q}^{m+1} f(z)-R_{q}^{m} f(z)\right]\right\} \\
& =1-\sum_{n=2}^{\infty}[n]_{q}^{m}\left([n]_{q}(L-1)-L\right) a_{n} z^{n-1} \\
& =1-\sum_{n=2}^{\infty}[n]_{q}^{m} \frac{[n]_{q}\left(e^{-i \theta}+B\right)-e^{-i \theta}+(B-A) \cos \lambda e^{-i \lambda}-B}{(A-B) \cos \lambda e^{-i \lambda}} a_{n} z^{n-1}
\end{aligned}
$$

Thus, the proof is completed.

Taking $q \rightarrow 1^{-}$in Theorem 5 , we get the following result.

Corollary 6. A necessary and sufficient condition for the function $f$ defined by (1) is in the class $\mathcal{S}^{m, \lambda}[A, B]$ is that

$$
1-\sum_{n=2}^{\infty} n^{m} \frac{n\left(e^{-i \theta}+B\right)-e^{-i \theta}+(B-A) \cos \lambda e^{-i \lambda}-B}{(A-B) \cos \lambda e^{-i \lambda}} a_{n} z^{n-1} \neq 0 .
$$

We next determine coefficient estimate for a function of form (1) to be in the class $\mathcal{S}_{q}^{m, \lambda}[A, B]$.

Theorem 7. If the function $f$ defined by (1) satisfies the following inequality

$$
\sum_{n=2}^{\infty}[n]_{q}^{m}\left\{[n]_{q}(1-B)-1+(A-B) \cos \lambda+B\right\}\left|a_{n}\right| \leq(A-B) \cos \lambda,
$$

then $f \in \mathcal{S}_{q}^{m, \lambda}[A, B]$. 
Proof. From Theorem 5, we write

$$
\begin{aligned}
& \left|1-\sum_{n=2}^{\infty}[n]_{q}^{m} \frac{[n]_{q}\left(e^{-i \theta}+B\right)-e^{-i \theta}+(B-A) \cos \lambda e^{-i \lambda}-B}{(A-B) \cos \lambda e^{-i \lambda}} a_{n} z^{n-1}\right| \\
& >1-\sum_{n=2}^{\infty}\left|[n]_{q}^{m} \frac{[n]_{q}\left(e^{-i \theta}+B\right)-e^{-i \theta}+(B-A) \cos \lambda e^{-i \lambda}-B}{(A-B) \cos \lambda e^{-i \lambda}}\right|\left|a_{n}\right| \\
& \geq 1-\sum_{n=2}^{\infty}[n]_{q}^{m} \frac{[n]_{q}(1-B)-1+\left|(A-B) \cos \lambda e^{-i \lambda}\right|+B}{\left|(A-B) \cos \lambda e^{-i \lambda}\right|}\left|a_{n}\right| \\
& =1-\sum_{n=2}^{\infty}[n]_{q}^{m} \frac{[n]_{q}(1-B)-1+(A-B) \cos \lambda+B}{(A-B) \cos \lambda}\left|a_{n}\right|>0,
\end{aligned}
$$

then $f \in \mathcal{S}_{q}^{m, \lambda}[A, B]$.

Corollary 8. Taking $q \rightarrow 1^{-}$in Theorem 7, we obtain

$$
\sum_{n=2}^{\infty} n^{m}\{n(1-B)-1+(A-B) \cos \lambda+B\}\left|a_{n}\right| \leq(A-B) \cos \lambda
$$

then $f \in \mathcal{S}^{m, \lambda}[A, B]$.

\section{REFERENCES}

[1] Andrews, G. E., Applications of basic hypergeometric functions, SIAM Rev. 16 (1974), 441484.

[2] Çaglar, M. and Deniz, E., Initial coefficients for a subclass of bi-univalent functions defined by Salagean differential operator, Commun. Fac. Sci. Univ. Ank. Ser. A1 Math. Stat. 66 (1) (2017), 85-91.

[3] Fine, N. J., Basic hypergeometric series and applications, Math. Surveys Monogr. 1988.

[4] Gasper, G. and Rahman, M., Basic hypergeometric series, Cambridge University Press, 2004.

[5] Goodman, A. W., Univalent functions, Volume I and Volume II, Mariner Pub. Co. Inc. Tampa Florida, 1984.

[6] Jackson, F. H., On $q$ - functions and a certain difference operator, Trans. Royal Soc. Edinburgh, 46 (1909), 253-281.

[7] Jackson, F. H., q- difference equations, Amer. J. Math. 32 (1910), 305-314.

[8] Janowski, W., Some extremal problems for certain families of analytic Functions I, Ann. Polon. Math. 28 (1973), 297-326.

[9] Kac, V. and Cheung, P., Quantum calculus, Springer, 2002.

[10] Salagean, G. S., Subclass of univalent functions, Complex Analysis-Fifth Romanian Finish Seminar, Bucharest, 1 (1998), 362-372.

Current address: Asena Çetinkaya: Department of Mathematics and Computer Sciences, Istanbul Kültür University, Istanbul, Turkey.

E-mail address: asnfigen@hotmail.com

ORCID Address: http://orcid.org/0000-0002-8815-5642 